\title{
VALORACIÓN DE LA SATISFACCIÓN DE LOS PACIENTES INGRESADOS EN OTORRINOLARINGOLOGÍA MEDIANTE LA ESCALA SERVQHOS
}

\section{Valuation of the satisfaction of patients admitted to the Otorhinolaryngology using the SERVQHOS questionnaire}

\author{
Estela PISÓN-CÁRCAMO; Pedro DÍAZ DE CERIO-CANDUELA \\ Servicio de Otorrinolaringología. Hospital San Pedro. Logroño. España \\ Correspondencia:pdiazcerio@gmail.com
}

Fecha de recepción: 7 de septiembre de 2018

Fecha de aceptación: 2 de octubre de 2018

Fecha de publicación: 5 de octubre de 2018

Fecha de publicación del fascículo: 1 de junio de 2019

Conflicto de intereses: Los autores declaran no tener conflictos de intereses

Imágenes: Los autores declaran haber obtenido las imágenes con el permiso de los pacientes

Política de derechos y autoarchivo: se permite el autoarchivo de la versión post-print (SHERPA/RoMEO)

Licencia CC BY-NC-ND. Licencia Creative Commons Atribución-NoComercial-SinDerivar 4.0 Internacional

Universidad de Salamanca. Su comercialización está sujeta al permiso del editor

RESUMEN: Objetivo: Evaluar la satisfacción de los pacientes, como medida de la calidad asistencial. Material y métodos: Estudio realizado desde febrero hasta julio del 2017. A todos los pacientes hospitalizados (187) se entregó al alta, un cuestionario anónimo y voluntario, basado en el modelo SERVQHOS, modificado y adaptado a dicho servicio. Resultados: Se recogieron 119 encuestas. La satisfacción media global (SERVQHOS): 4,2 con desviación estándar (DE): $\pm 0,67$. Los aspectos mejor valorados: el trato personalizado y la amabilidad del personal. Los aspectos peor valorados: las indicaciones y la facilidad para llegar al hospital, siendo los únicos aspectos que no evidenciaron tener relación con la satisfacción global (Correlación de Pearson, valor de p>0,05). El 95,8\% se mostraron muy satisfechos o satisfechos con los cuidados recibidos y el 98,3\% recomendaría el hospital. En relación con las variables sociodemográficas estudiadas, se encontró significación estadística en la variable edad con el nivel de satisfacción global (Chi-2, valor de $\mathrm{p}=0,021$ ). De forma general, las personas más satisfechas fueron aquellas con una edad comprendida entre los 46-77 años, sexo masculino, casados, sin ocupación laboral y con nivel de estudios superiores. Por último, se encontró significación estadística en las variables sexo y estado civil con algunos de los aspectos asistenciales valorados con los 19 ítems. Conclusiones: La mayoría de los pacientes se mostraron satisfechos 


\section{VALORACIÓN DE LA SATISFACCIÓN DE LOS PACIENTES INGRESADOS EN OTORRINOLARINGOLOGÍA MEDIANTE LA ESCALA SERVQHOS PISÓN-CÁRCAMO E y DÍAZ DE CERIO-CANDUELA P}

en todas las dimensiones exploradas y casi la totalidad recomendarían este hospital, evidenciando que la satisfacción está muy ligada a las cualidades y competencias del personal en su relación con el paciente.

PALABRAS CLAVE: satisfacción del paciente; encuesta; calidad asistencial; otorrinolaringología

SUMMARY: Objective: To evaluate the satisfaction of patients, as a measure of the quality of care. Material and methods: Study carried out from February to August 2017. All the hospitalized patients (187) were given at the time of discharge an anonymous and voluntary questionnaire, based on the SERVQHOS model, modified and adapted to that Department. Results: A total of 119 questionnaires were collected. The overall average satisfaction (SERVQHOS): 4.2 with standard deviation (SD): \pm 0.67 . The most valued aspects: the personalized treatment and the friendliness of the staff. The least valued aspects: the indications and the ease of getting to the hospital, being the only aspects that did not show any relationship with the level of global satisfaction (Pearson correlation, value of $\mathrm{p}>0,05)$. The large majority $(95.8 \%)$ were very satisfied or satisfied with the health care received and $98.3 \%$ would recommend the hospital. In relation to the sociodemographic variables studied, statistical significance was found in the age variable regarding the level of overall satisfaction (Chi-2, $\mathrm{p}=0.021)$. In general, the most satisfied people were those between the ages of 46-77, male, married, with no occupation and with a higher education level. Finally, statistical significance was found in the demographic variables sex and marital status with respect to some of the aspects of care assessed with the 19 items. Conclusion: Most patients were satisfied in all the dimensions explored and the vast majority would recommend this hospital, showing that satisfaction is closely linked to the qualities and skills of staff in their relationship with the patient.

KEYWORDS; patient satisfaction; survey; quality of care; otorhinolaryngology.

\section{INTRODUCCIÓN}

La satisfacción del paciente ha adquirido una gran importancia y su valor va en aumento en todas las organizaciones, siendo en la actualidad una pieza angular de la calidad asistencial [1]. En los servicios sanitarios, se hace necesaria una mejora continua de la calidad para lo que es importante conocer la visión de sus pacientes [2]. De esta forma el ciudadano se convierte en el motor del sistema sanitario. Las necesidades del paciente son el eje sobre el que se deben articular las prestaciones asistenciales y construir la base de la organización de los servicios y de los propios hospitales [3].

El Modelo EFQM de Excelencia (Fundación Europea para la Gestión de Calidad) [4] recoge en su criterio de resultados, la recomendación de evaluar sistemáticamente la percepción que tienen los usuarios de las organizaciones y establecer medidas internas para predecir y mejorar la calidad de los servicios [5].
En este contexto, el III Plan de Salud de La Rioja, comunidad autónoma donde se realiza el estudio, recoge entre sus objetivos optimizar resultados de salud mediante una práctica asistencial centrada en las necesidades reales del paciente, promoviendo la realización de encuestas de satisfacción y estableciendo planes de mejora a partir de las mismas [6].

Existen diferentes modelos de evaluación de la calidad percibida basados en encuestas, las cuales constituyen la herramienta más sencilla para conocer cómo evalúan los pacientes el servicio recibido y establecer una retroalimentación informativa hacia los profesionales a fin de que puedan modificar todos aquellos aspectos deficientemente percibidos y evaluar los resultados del cuidado [7].

En este estudio se utilizó el modelo de encuesta de calidad de la asistencia sanitaria SERVQHOS [8], que es una adaptación al ámbito hospitalario en España de la encuesta SERVQHAL [9], diseñada 


\section{VALORACIÓN DE LA SATISFACCIÓN DE LOS PACIENTES INGRESADOS EN OTORRINOLARINGOLOGÍA MEDIANTE LA ESCALA SERVQHOS PISÓN-CÁRCAMO E y DÍAZ DE CERIO-CANDUELA P}

por Parasuraman, Zeithaml y Berri (1985), y reconocida como un instrumento sólido para detectar la calidad percibida en empresas de servicios [10]. Su composición valora, por un lado, las expectativas sobre el servicio ideal y, por otro, las percepciones de los usuarios del servicio que han recibido se basan por tanto en el paradigma de la desconfirmación [11-13].

\section{MATERIAL Y MÉTODO}

Se trata de un estudio transversal basado en una encuesta de satisfacción realizada a los pacientes ingresados en el Servicio de Otorrinolaringología y Cirugía de Cabeza y Cuello, del Hospital San Pedro de La Rioja, desde el 17/02/2017 al $31 / 07 / 2017$, que permitió conocer los aspectos del proceso asistencial que contribuyen a la satisfacción e insatisfacción del paciente, analizar la asociación entre el nivel de satisfacción y las características sociodemográficas de los pacientes, y determinar el peso específico de cada uno de los ítems de la encuesta con respecto a la satisfacción global.

CRITERIOS DE SELECCIÓN. Se incluyeron en el estudio aquellos pacientes mayores o iguales de 14 años, que aceptaron participar de manera anónima $\mathrm{y}$ voluntaria.

Instrumento DE MEDidA. Se elaboró una encuesta de medición de satisfacción basada en el modelo SERVQHOS [9], modificada y adaptada a los pacientes ingresados en dicho servicio. Se configuró en un único folio por ambas caras. Previamente se enunciaban las instrucciones correspondientes a cada apartado, para su correcta cumplimentación.

El anverso constaba de:

- Una introducción indicando el objetivo del estudio, donde se especificaba que la participación era voluntaria y anónima, garantizando la confidencialidad de los datos.

- Un primer bloque relativo a la asistencia sanitaria, basado en la escala de satisfacción SERVQHOS [9], validada en España en el ámbito hospitalario. Se aplicó la escala original, con dos modificaciones. Se eliminó el ítem "puntualidad en la consulta», puesto que no procede en una planta de hospitalización. Y se añadió el ítem «el interés del personal médico por los pacientes ha sido», porque en la escala original sólo se incluye «el interés del personal de enfermería por los pacientes ha sido», siendo ambos profesionales los responsables de la atención y de la calidad asistencial. Quedando configurada con 19 ítems puntuados en una escala Likert del 1 («la asistencia recibida ha sido mucho peor de lo que esperaba») al 5 («la asistencia sanitaria ha sido mucho mejor de lo esperado»). De esta manera las puntuaciones más altas hacían referencia a aspectos que los pacientes valoraban positivamente y que resultaron ser mejor de lo que esperaban.

- Un segundo bloque con criterios adicionales de referencia que complementaban el apartado anterior, compuesto por seis preguntas independientes con dos alternativas de respuesta («si/no»), y una última cuestión directa sobre la satisfacción global con cuatro alternativas de respuesta («nada satisfecho, poco satisfecho, satisfecho, muy satisfecho»).

El reverso constaba de:

- Un primer bloque donde se solicitaba información demográfica («edad, sexo, estado civil, situación actual y estudios concluidos»).

- Un segundo bloque con un espacio libre para que el usuario expresase sus opiniones de mejora.

- Para finalizar, se incluyó una frase de agradecimiento a la participación prestada.

Proceso de ReCOGIDA DE INFORMACIÓN. El personal de enfermería de la unidad se encargó de entregar los cuestionarios, solicitando a los pacientes su participación en el estudio. Se determinó que la entrega de las encuestas se haría de forma consecutiva a todo paciente dado de alta, durante el periodo de estudio. Tras cumplimentar 


\section{VALORACIÓN DE LA SATISFACCIÓN DE LOS PACIENTES INGRESADOS EN OTORRINOLARINGOLOGÍA MEDIANTE LA ESCALA SERVQHOS PISÓN-CÁRCAMO E y DÍAZ DE CERIO-CANDUELA P}

los cuestionarios de manera anónima y voluntaria, los pacientes los depositaban posteriormente en el buzón ubicado en el control de enfermería del servicio.

AnÁlisis estadístico. El análisis estadístico se realizó con el programa estadístico SPSS 24. Los datos cuantitativos se presentan como medias con la desviación típica. Los datos cualitativos en forma de proporciones. A lo largo de todo el estudio se consideró significación estadística los valores de $\mathrm{p}$ $<0,05$ e intervalos de confianza al 95\%.

\section{RESULTADOS}

De 187 pacientes ingresados sólo 119 respondieron la encuesta para una participación del 63.6\%.

La distribución de la muestra según variables sociodemográficas se presenta en la Tabla 1 . Siendo el factor edad muy amplio, se decidió trabajar por agrupamientos. De los 119 encuestados 69 eran hombres y 48 mujeres, con una edad media de $51,23 \pm 18$ años. La mayor parte estaban casados $60,5 \%$. El $41,2 \%$ se encontraban en activo y el $33,6 \%$ eran jubilados. El 2,5\% no tenían ningún estudio y el resto refirió haber completado algún tipo de estudios.

El análisis de las medias de las variables evaluadas en la primera parte del cuestionario evidenció que la mayoría de los pacientes se encontraron satisfechos en todas las dimensiones exploradas con una puntuación superior a 3,5 (en una escala del 1 al 5 , donde 1 es «mucho peor de lo que esperaba» y 5 «mucho mejor de lo que esperaba»), por lo que todos los ítems se valoraron según las expectativas o por encima de éstas. Los aspectos con los que los pacientes se mostraron más satisfechos fueron «el trato personalizado recibido» $(4,53 \pm 0,701)$ y «la amabilidad del personal en su trato con la gente» $(4,51 \pm 0,687)$, consiguiendo ambas una puntuación por encima de 4,5 , que corresponde a las categorías «mejor de lo que se esperaba». Los aspectos peor valorados fueron «la facilidad para llegar al hospital» $(3,79 \pm 0,972) \mathrm{y}$ «las indicaciones para llegar al servicio de otorrinolaringología» $(3,98 \pm 0,924)$. La puntuación media de los 19 ítems sobre la calidad asistencial percibida fue de 4,2 $\pm 0,67$ (Tabla 2).

Tabla 1. Características sociodemográficas de los pacientes que respondieron a la encuesta.

\begin{tabular}{|c|c|c|c|}
\hline $\begin{array}{c}\text { Características } \\
\text { Sociodemográficas }\end{array}$ & $\begin{array}{l}\text { Número } \\
\text { (n) }\end{array}$ & $\begin{array}{l}\text { Porcentaje } \\
(\%)\end{array}$ & $\begin{array}{c}\text { No } \\
\text { contestan }\end{array}$ \\
\hline $\begin{array}{l}\text { Edad recodificada } \\
14-29 \text { años } \\
30-45 \text { años } \\
46-60 \text { años } \\
61-77 \text { años } \\
\text { 78-89 años } \\
\text { Edad media (años) } \\
51,23 \pm 18\end{array}$ & $\begin{array}{c}17 \\
22 \\
37 \\
34 \\
5\end{array}$ & $\begin{array}{c}14,3 \\
18,5 \\
31,1 \\
28,6 \\
4,2\end{array}$ & 4 \\
\hline $\begin{array}{l}\text { Sexo } \\
\text { Hombre } \\
\text { Mujer }\end{array}$ & $\begin{array}{l}69 \\
48\end{array}$ & $\begin{array}{c}58 \\
40,3\end{array}$ & 2 \\
\hline $\begin{array}{l}\text { Estado civil } \\
\text { Soltero } \\
\text { Casado } \\
\text { Viudo } \\
\text { Separado }\end{array}$ & $\begin{array}{c}29 \\
72 \\
5 \\
10\end{array}$ & $\begin{array}{c}24,4 \\
60,5 \\
4,2 \\
8,4\end{array}$ & 3 \\
\hline $\begin{array}{l}\text { Situación laboral } \\
\text { actual } \\
\text { Jubilado } \\
\text { En desempleo } \\
\text { Trabajando } \\
\text { Ama/o de casa } \\
\text { Estudiante }\end{array}$ & $\begin{array}{c}40 \\
9 \\
49 \\
7 \\
10\end{array}$ & $\begin{array}{c}33,6 \\
7,6 \\
41,2 \\
5,9 \\
8,4\end{array}$ & 4 \\
\hline $\begin{array}{l}\text { Estudios concluidos } \\
\text { Sin estudios } \\
\text { Primarios } \\
\text { Bachiller } \\
\text { Universitarios }\end{array}$ & $\begin{array}{c}3 \\
52 \\
31 \\
28\end{array}$ & $\begin{array}{c}2,5 \\
43,7 \\
26,1 \\
23,5\end{array}$ & 5 \\
\hline
\end{tabular}

Posteriormente, teniendo en cuenta el tamaño de la muestra (119 sujetos) y que algunas opciones de respuesta recogen menos del $5 \%$ de la información, para evitar sesgos de respuestas extremas y errores en las estimaciones de las predicciones, se procedió a recodificar las alternativas de respuesta, en una escala del 1 al 3 (donde 1 incluye «mucho peor y peor de lo esperado», 2 se corresponde con «lo esperado» y 3 con «mejor y mucho mejor de 


\section{VALORACIÓN DE LA SATISFACCIÓN DE LOS PACIENTES INGRESADOS EN OTORRINOLARINGOLOGÍA MEDIANTE LA ESCALA SERVQHOS PISÓN-CÁRCAMO E y DÍAZ DE CERIO-CANDUELA P}

Tabla 2. Calidad de la asistencia sanitaria percibida en los 19 ítems del primer bloque de la encuesta.

\begin{tabular}{|c|c|c|c|c|c|c|c|}
\hline & $\begin{array}{c}\text { Mucho } \\
\text { peor } \\
\text { de lo } \\
\text { esperado } \\
\text { n (\%) } \\
1\end{array}$ & $\begin{array}{c}\text { Peor de lo } \\
\text { esperado } \\
\text { n (\%) } \\
\\
2\end{array}$ & $\begin{array}{c}\text { Lo } \\
\text { esperado } \\
\text { n (\%) } \\
\\
3\end{array}$ & $\begin{array}{c}\text { Mejor de lo } \\
\text { esperado } \\
\text { n (\%) } \\
4\end{array}$ & $\begin{array}{c}\text { Mucho } \\
\text { mejor de lo } \\
\text { esperado } \\
\text { n }(\%) \\
5\end{array}$ & $\begin{array}{c}\text { No } \\
\text { contesta } \\
\mathrm{n}(\%)\end{array}$ & $\begin{array}{c}\text { Media } \\
\text { (D.típica) }\end{array}$ \\
\hline La facilidad para llegar al hospital & $3(2,5 \%)$ & $2(1,7 \%)$ & $46(38,7 \%)$ & $32(26,9 \%)$ & $34(28,6 \%)$ & 2 & $\begin{array}{c}3,79 \\
(0,972)\end{array}$ \\
\hline $\begin{array}{l}\text { Las indicaciones para llegar al } \\
\text { Servicio de Otorrinolaringología }\end{array}$ & $1(0,8 \%)$ & $2(1,7 \%)$ & $39(32,8 \%)$ & $32(26,9 \%)$ & $44(37 \%)$ & 1 & $\begin{array}{c}3,98 \\
(0,924)\end{array}$ \\
\hline El estado de las habitaciones & $1(0,8 \%)$ & $2(1,7 \%)$ & $35(29,4 \%)$ & $35(29,4 \%)$ & $46(38,7 \%)$ & 0 & $\begin{array}{c}4,03 \\
(0,911)\end{array}$ \\
\hline $\begin{array}{l}\text { La tecnología de los equipos médicos } \\
\text { utilizados }\end{array}$ & $2(1,7 \%)$ & 0 & $22(18,5 \%)$ & $40(33,6 \%)$ & $55(46,2 \%)$ & 0 & $\begin{array}{c}4,13 \\
(0,849) \\
\end{array}$ \\
\hline La apariencia del personal sanitario & $1(0,8 \%)$ & 0 & $13(10,9 \%)$ & $37(31,1 \%)$ & $68(57,1 \%)$ & 0 & $\begin{array}{c}4,26 \\
(0,807)\end{array}$ \\
\hline $\begin{array}{l}\text { La preparación del personal para } \\
\text { realizar su trabajo }\end{array}$ & $1(0,8 \%)$ & 0 & $21(17,6 \%)$ & $42(35,3 \%)$ & $55(46,2 \%)$ & 0 & $\begin{array}{c}4,31 \\
(0,821)\end{array}$ \\
\hline $\begin{array}{l}\text { El tiempo de espera para ser } \\
\text { atendido por el personal sanitario }\end{array}$ & $3(2,5 \%)$ & $4(3,4 \%)$ & $21(17,6 \%)$ & $38(31,9 \%)$ & $53(44,5 \%)$ & 0 & $\begin{array}{c}4,13 \\
(0,988) \\
\end{array}$ \\
\hline $\begin{array}{l}\text { La información que los médicos } \\
\text { proporcionan al paciente }\end{array}$ & $2(1,7 \%)$ & 0 & $22(18,5 \%)$ & $40(33,6 \%)$ & $55(46,2 \%)$ & 0 & $\begin{array}{c}4,23 \\
(0,868) \\
\end{array}$ \\
\hline $\begin{array}{l}\text { La información que los médicos } \\
\text { proporcionan a familiares }\end{array}$ & $2(1,7 \%)$ & $2(1,7 \%)$ & $27(22,7 \%)$ & $36(30,3 \%)$ & $52(43,7 \%)$ & 0 & $\begin{array}{c}4,13 \\
(0,935) \\
\end{array}$ \\
\hline $\begin{array}{l}\text { La disposición del personal para } \\
\text { ayudarle cuando lo necesite }\end{array}$ & $1(0,8 \%)$ & 0 & $13(10,9 \%)$ & $37(31,1 \%)$ & $68(57,1 \%)$ & 0 & $\begin{array}{c}4,44 \\
(0,755) \\
\end{array}$ \\
\hline $\begin{array}{l}\text { La rapidez con que consigue lo que } \\
\text { necesita o pide }\end{array}$ & 0 & $2(1,7 \%)$ & $14(11,8 \%)$ & $41(34,5 \%)$ & $62(52,1 \%)$ & 0 & $\begin{array}{c}4,37 \\
(0,758) \\
\end{array}$ \\
\hline $\begin{array}{l}\text { La capacidad del personal para } \\
\text { comprender sus necesidades }\end{array}$ & 0 & 0 & $19(16 \%)$ & $36(30,3 \%)$ & $64(53,8 \%)$ & 0 & $\begin{array}{c}4,38 \\
(0,748) \\
\end{array}$ \\
\hline $\begin{array}{l}\text { El interés del personal por solucionar } \\
\text { sus problemas }\end{array}$ & 0 & 0 & $21(17,6 \%)$ & $31(26,1 \%)$ & $66(55,5 \%)$ & 1 & $\begin{array}{c}4,38 \\
(0,773) \\
\end{array}$ \\
\hline $\begin{array}{l}\text { El interés del personal por cumplir lo } \\
\text { que promete }\end{array}$ & 0 & 0 & $26(21,8 \%)$ & $31(26,1 \%)$ & $61(51,3 \%)$ & 1 & $\begin{array}{c}4,30 \\
(0,8099 \\
\end{array}$ \\
\hline $\begin{array}{l}\text { El interés del personal médico por el } \\
\text { paciente }\end{array}$ & 0 & 0 & $19(16 \%)$ & $29(24,4 \%)$ & $70(58,8 \%)$ & 1 & $\begin{array}{c}4,43 \\
(0,756)\end{array}$ \\
\hline $\begin{array}{l}\text { El interés del personal de enfermería } \\
\text { por el paciente }\end{array}$ & 0 & 0 & $16(13,4 \%)$ & $33(27,7 \%)$ & $70(58,8 \%)$ & 0 & $\begin{array}{c}4,45 \\
(0,722) \\
\end{array}$ \\
\hline $\begin{array}{l}\text { La amabilidad del personal en su } \\
\text { trato con la gente }\end{array}$ & 0 & 0 & $13(10,9 \%)$ & $32(26,9 \%)$ & $74(62,2 \%)$ & 0 & $\begin{array}{c}4,51 \\
(0,687) \\
\end{array}$ \\
\hline $\begin{array}{l}\text { La confianza que el personal le ha } \\
\text { transmitido }\end{array}$ & 0 & 0 & $16(13,4 \%)$ & $34(28,6 \%)$ & $68(57,1 \%)$ & 1 & $\begin{array}{c}4,44 \\
(0,723) \\
\end{array}$ \\
\hline $\begin{array}{l}\text { El trato personalizado que ha } \\
\text { recibido }\end{array}$ & 0 & 0 & $14(11,8 \%)$ & $28(23,5 \%)$ & $76(63,9 \%)$ & 1 & $\begin{array}{c}4,53 \\
(0,701)\end{array}$ \\
\hline
\end{tabular}




\section{VALORACIÓN DE LA SATISFACCIÓN DE LOS PACIENTES INGRESADOS EN OTORRINOLARINGOLOGÍA MEDIANTE LA ESCALA SERVQHOS PISÓN-CÁRCAMO E y DÍAZ DE CERIO-CANDUELA P}

lo esperado»). Tras la recodificación, el ítem más valorado fue «la amabilidad del personal en su trato con la gente» $(2,89 \pm 0,313)$ y el segundo «el trato personalizado recibido» $(2,88 \pm 0,325)$.

Analizando las preguntas dicotómicas del segundo bloque (Figura 1), el 60,5\% conocían el nombre del médico que les atendía, sin embargo, sólo un $27,7 \%$ de los encuestados conocían el nombre de la enfermera que les atendía. Por otra parte, casi la mayor parte de la muestra, el 97,5\% contestaron que no le realizaron en el hospital ninguna prueba sin su consentimiento, el $92,4 \%$ afirmaron que si recibieron suficiente información sobre su enfermedad y el $95 \%$ opinaron haber estado ingresado el tiempo necesario. Por último, el 98,3\% recomendarían este hospital a otras personas, mientras que el 1,7\% restantes no contestaron a este apartado.

En relación con la segunda parte del cuestionario a la pregunta realizada sobre el nivel de satisfacción global, un 76,5\% estaban muy satisfechos, un $19,3 \%$ estaban satisfechos y $4,2 \%$ estaban poco satisfechos. Nadie estuvo nada satisfecho.

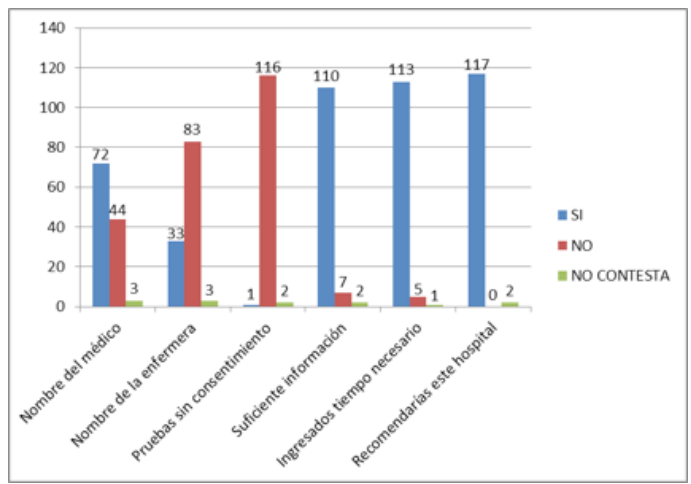

Figura 1. Respuesta a las preguntas dicotómicas incluidas en el segundo bloque de la encuesta.
El análisis de la correlación entre las variables del primer bloque del cuestionario (19 ítem) con la satisfacción global, demostró que todos estaban relacionados con la satisfacción $(\mathrm{p}<0,05)$, excepto 2 de los ítems: «la facilidad para llegar al hospital» (valor de $\mathrm{p}>0,05$ ) y «las indicaciones para llegar al servicio de otorrinolaringología» (valor de $\mathrm{p}>$ 0,05), (Tabla 3 ).

En relación a las variables sociodemográficas de los pacientes, sólo se encontró significación estadística en la variable edad al estudiar su influencia con la satisfacción global percibida $($ Chi-2 $<0,05)$. Se realizó un segundo análisis tras agrupar no sólo la edad, sino también el resto de las variables sociodemográficas. No hubo diferencias. (Tabla 4). Se observó que las personas que se encontraron más satisfechas fueron aquellas con una edad comprendida entre los 46-77 años, sexo masculino, casados, sin ocupación laboral y con estudios superiores.

Por último, se procedió a valorar las variables sociodemográficas de los pacientes relacionadas con cada aspecto asistencial del primer bloque (19 ítems), ambas recodificadas, para agrupar los datos y alternativas de respuestas. (Tabla 5). Se encontró significación estadística en la variable demográfica sexo con respecto a 3 ítems «la capacidad del personal para comprender sus necesidades» (Chi-2 de 0,022 ), «la amabilidad del personal con el trato con la gente» (Chi-2 de 0,046), y el ítem «el trato personalizado que ha recibido» (Chi-2 de 0,033), donde las mujeres puntuaron estos ítems mejor que los hombres. En cuanto a la variable estado civil, se observaron diferencias significativas con respecto al ítem «el tiempo de espera para ser atendido por el personal sanitario " (Chi-2 de 0,046), donde el $93,6 \%$ de los casados puntuaron mejor este ítem. El resto de las variables sociodemográficas recodificadas no explican de manera significativa el comportamiento del resto de los 19 ítems recodificados. 


\section{VALORACIÓN DE LA SATISFACCIÓN DE LOS PACIENTES INGRESADOS EN OTORRINOLARINGOLOGÍA MEDIANTE LA ESCALA SERVQHOS PISÓN-CÁRCAMO E y DÍAZ DE CERIO-CANDUELA P}

Tabla 3. Variables del primer apartado de la encuesta SERVQHOS (19 ítems) que se correlacionan significativamente con el nivel de satisfacción global de los cuidados recibidos durante la estancia de los pacientes en el hospital.

\begin{tabular}{|l|c|c|}
\hline \multicolumn{1}{|c|}{ Variables } & Correlación de Pearson & $p$ \\
\hline La facilidad para llegar al hospital & 0,180 & 0,180 \\
Las indicaciones para llegar al Servicio de Otorrinolaringología & 0,117 & 0,104 \\
El estado de las habitaciones & 0,210 & 0,011 \\
La tecnología de los equipos médicos utilizados & 0,264 & 0,002 \\
La apariencia del personal sanitario & 0,266 & 0,002 \\
La preparación del personal para realizar su trabajo & 0,236 & 0,005 \\
El tiempo de espera para ser atendido por el personal sanitario & 0,307 & 0,000 \\
La información que los médicos proporcionan al paciente & 0,173 & 0,030 \\
La información que los médicos proporcionan a familiares & 0,257 & 0,002 \\
La disposición del personal para ayudarle cuando lo necesite & 0,302 & 0,000 \\
La rapidez con que consigue lo que necesita o pide & 0,338 & 0,000 \\
La capacidad del personal para comprender sus necesidades & 0,349 & 0,001 \\
El interés del personal por solucionar sus problemas & 0,280 & 0,001 \\
El interés del personal por cumplir lo que promete & 0,292 & 0,001 \\
El interés del personal médico por el paciente & 0,295 & 0,000 \\
El interés del personal de enfermería por el paciente & 0,328 & 0,003 \\
La amabilidad del personal en su trato con la gente & 0,251 & 0,000 \\
La confianza que el personal le ha transmitido & 0,342 & 0,011 \\
El trato personalizado que ha recibido & 0,212 & \\
\hline
\end{tabular}

Tabla 4. Análisis de la influencia de los datos sociodemográficos recodificados con la satisfacción global del paciente.

\begin{tabular}{|c|c|c|c|c|}
\hline & $\begin{array}{c}\text { Poco satisfecho } \\
\mathrm{n}(\%)\end{array}$ & $\begin{array}{c}\text { Satisfecho } \\
\mathrm{N}(\%)\end{array}$ & $\begin{array}{c}\text { Muy satisfecho } \\
\mathrm{n}(\%)\end{array}$ & $\begin{array}{c}\text { Test } \\
\text { Chi-2(p) }\end{array}$ \\
\hline $\begin{array}{l}\text { Edad recodificada } \\
14-29 \text { años } \\
\text { 30-45 años } \\
46-60 \text { años } \\
61-77 \text { años } \\
78-88 \text { años }\end{array}$ & $\begin{array}{c}2(11,8 \%) \\
0(0 \%) \\
1(2,7 \%) \\
2(5,9 \%) \\
0(\%)\end{array}$ & $\begin{array}{c}8(47,1 \%) \\
5(22,7 \%) \\
4(10,8 \%) \\
3(8,8 \%) \\
1(20 \%)\end{array}$ & $\begin{array}{c}7(41,2 \%) \\
17(77,3 \%) \\
32(86,5 \%) \\
29(85,3 \%) \\
4(80 \%)\end{array}$ & 0,021 \\
\hline $\begin{array}{l}\text { Sexo } \\
\text { Hombre } \\
\text { Mujer } \\
\end{array}$ & $\begin{array}{l}4(5,8 \%) \\
1(2,1 \%)\end{array}$ & $\begin{array}{l}11(15,9 \%) \\
11(22,9 \%)\end{array}$ & $\begin{array}{c}54(78,3 \%) \\
36(75 \%)\end{array}$ & 0,431 \\
\hline $\begin{array}{l}\text { Estado civil } \\
\text { No casados (solteros, viudos, separados) } \\
\text { Casados }\end{array}$ & $\begin{array}{l}3(6,8 \%) \\
2(2,8 \%)\end{array}$ & $\begin{array}{c}9(20,5 \%) \\
13(18,1 \%)\end{array}$ & $\begin{array}{l}32(72,7 \%) \\
57(79,2 \%)\end{array}$ & 0,531 \\
\hline $\begin{array}{l}\text { Situación laboral actual } \\
\text { Trabajando (trabajando, amo/a de casa) } \\
\text { Sin trabajar (en desempleo, estudiante) }\end{array}$ & $\begin{array}{l}1(1,8 \%) \\
4(6,8 \%)\end{array}$ & $\begin{array}{c}12(21,4 \%) \\
9(15 \%)\end{array}$ & $\begin{array}{c}43(76,8 \%) \\
46(78 \%)\end{array}$ & 0,324 \\
\hline $\begin{array}{l}\text { Estudios concluidos } \\
\text { Hasta primarios (sin estudios, primarios) } \\
\text { Superiores (bachiller, universitarios) }\end{array}$ & $\begin{array}{l}3(5,5 \%) \\
2(3,4 \%)\end{array}$ & $\begin{array}{c}9(16,4 \%) \\
12(20,3 \%)\end{array}$ & $\begin{array}{l}43(78,2 \%) \\
45(76,3 \%)\end{array}$ & 0,766 \\
\hline
\end{tabular}




\section{VALORACIÓN DE LA SATISFACCIÓN DE LOS PACIENTES INGRESADOS EN OTORRINOLARINGOLOGÍA MEDIANTE LA ESCALA SERVQHOS PISÓN-CÁRCAMO E y DÍAZ DE CERIO-CANDUELA P}

Tabla 5. Análisis de la influencia de los 19 ítems recodificados con las variables demográficas recodificadas (test Chi-2).

\begin{tabular}{|l|c|c|c|c|c|}
\hline \multicolumn{1}{|c|}{$\begin{array}{c}\text { Îtems recodificados } \\
\text { Alternativas de respuesta en escala del 1 a 3) }\end{array}$} & Edad & $\begin{array}{c}\text { Estado } \\
\text { civil }\end{array}$ & Laboral & Estudios & Sexo \\
\hline La facilidad para llegar al hospital & 0,752 & 0,673 & 0,281 & 0,719 & 0,531 \\
\hline Las indicaciones para llegar al Servicio de Otorrinolaringología & 0,822 & 0,956 & 0,598 & 0,064 & 0,957 \\
\hline El estado de las habitaciones & 0,322 & 0,969 & 0,197 & 0,227 & 0,932 \\
\hline La tecnología de los equipos médicos utilizados & 0,506 & 0,935 & 0,342 & 0,93 & 0,542 \\
\hline La apariencia del personal sanitario & 0,730 & 0,644 & 0,558 & 0,582 & 0,665 \\
\hline La preparación del personal para realizar su trabajo & 0,828 & 0,933 & 0,553 & 0,386 & 0,454 \\
\hline El tiempo de espera para ser atendido por el personal sanitario & 0,553 & 0,046 & 0,760 & 0,948 & 0,215 \\
\hline La información que los médicos proporcionan al paciente & 0,635 & 0,462 & 0,822 & 0,661 & 0,339 \\
\hline La información que los médicos proporcionan a familiares & 0,456 & 0,773 & 0,514 & 0,975 & 0,354 \\
\hline La disposición del personal para ayudarle cuando lo necesite & 0,943 & 0,617 & 0,583 & 0,613 & 0,256 \\
\hline La rapidez con que consigue lo que necesita o pide & 0,329 & 0,926 & 0,307 & 0,315 & 0,74 \\
\hline La capacidad del personal para comprender sus necesidades & 0,727 & 0,135 & 0,704 & 0,871 & 0,022 \\
\hline El interés del personal por solucionar sus problemas & 0,486 & 0,671 & 0,879 & 0,640 & 0,71 \\
\hline El interés del personal por cumplir lo que promete & 0,371 & 0,981 & 0,730 & 0,548 & 0,89 \\
\hline El interés del personal médico por el paciente & 0,919 & 0,957 & 0,276 & 0,901 & 0,385 \\
\hline El interés del personal de enfermería por el paciente & 0,974 & 0,969 & 0,334 & 0,880 & 0,161 \\
\hline La amabilidad del personal en su trato con la gente & 0,948 & 0,572 & 0,846 & 0,873 & 0,046 \\
\hline La confianza que el personal le ha transmitido & 0,769 & 0,946 & 0,880 & 0,727 & 0,056 \\
\hline El trato personalizado que ha recibido & 0,912 & 0,426 & 0,888 & 0,642 & 0,033 \\
\hline
\end{tabular}

\section{DISCUSIÓN}

El nivel de colaboración en la cumplimentación de la encuesta SERVQHOS fue del 63,6\%. Se desconocen los motivos por los que el $36,4 \%$ de pacientes no rellenaron la encuesta, pudiendo deberse entre otras causas, o bien que no quisieron participar o bien que no se les entregó la encuesta al alta solicitando su colaboración. En los estudios de ámbito general el índice de participación está alrededor del $30 \%$ y en los centrados en una población específica, como el nuestro, se obtienen porcentajes más elevados cercanos al 60\% [7,14,17,18]. Además, en general, en aquellos estudios donde la encuesta se entregó inmediatamente tras el alta hospitalaria como en este caso, presentan tasas de participación más altas $[8,14]$, que con otras formas de recogida como el envío por correo o el uso de buzones, que se asocian a participaciones más bajas (entre el 20 y el 30\%) $[16,19]$.

Los resultados obtenidos evidencian que los pacientes valoraron muy positivamente su paso por el servicio de otorrino. El 95,8\% estuvieron muy satisfechos o satisfechos con los cuidados sanitarios recibidos. Estos resultados son similares a estudios previos, donde se observa que la percepción que tienen los pacientes sobre los cuidados hospitalarios es buena $[8,14,15]$. Sin embargo, en nuestro estudio, no hubo ningún paciente insatisfecho, cuando en otros centros este dato suele oscilar del 0,2 al $3 \%[7,19]$.

En cuanto a la valoración de los 19 ítems sobre los diferentes aspectos de la asistencia sanitaria, primero se analizaron con 5 alternativas 


\section{VALORACIÓN DE LA SATISFACCIÓN DE LOS PACIENTES INGRESADOS EN OTORRINOLARINGOLOGÍA MEDIANTE LA ESCALA SERVQHOS PISÓN-CÁRCAMO E y DÍAZ DE CERIO-CANDUELA P}

de respuesta y posteriormente agrupados con 3 alternativas de respuesta, para evitar errores en las estimaciones de las predicciones, sin observarse diferencias estadísticamente significativas entre ambos análisis. Las puntuaciones de los 19 ítems fueron altas, evidenciando que la mayoría de los pacientes se encontraron satisfechos en todas las dimensiones exploradas. Estos resultados coinciden o son un poco superiores a algunos de los estudios revisados $[8,14]$.

Los aspectos de la asistencia sanitaria mejor puntuados, al igual que otros trabajos realizados, corresponden a «la amabilidad del personal en su trato con la gente» $\mathrm{y}$ «al trato personalizado recibido» $[8,14,15,20]$ (calidad subjetiva). Por el contrario, los aspectos peor puntuados fueron «la facilidad para llegar al hospital»y «las indicaciones para llegar al servicio de otorrinolaringología» (calidad objetiva). Por lo tanto, en general, el paciente se encuentra satisfecho con la atención que recibe, pero encuentra carencias en aspectos estructurales y de accesibilidad al hospital $[7,17]$.

Por otra parte, se evidenció que todos los aspectos estudiados estaban relacionados con la satisfacción global, excepto: «la facilidad para llegar al hospital»y «las indicaciones para llegar al servicio de otorrinolaringología», excepciones que no coinciden con otros estudios [7]. Se observó que las variables más predictoras de la satisfacción fueron «el tiempo de espera para ser atendido por el personal sanitario», «la disposición del personal para ayudarle cuando lo necesite», «la rapidez con que consigue lo que necesita», «la capacidad del personal para comprender sus necesidades» $\mathrm{y}$ «la confianza que el personal le ha trasmitido». La mayoría de estos aspectos vinculados con la relación profesional-paciente y que también fueron destacados en los comentarios aportados por algunos pacientes en el apartado observaciones al final del cuestionario.

Otros aspectos muy valorados fueron, «el interés del personal por solucionar problemas» $\mathrm{y}$ «el interés del personal por cumplir lo que promete».
Todos ellos implicados en el proceso de atención sanitaria con el usuario y con el trato del personal con los pacientes, al igual que en otros estudios $[7,14,17,21]$. Destaca "el interés del personal de enfermería por el paciente" y «el interés del personal médico por el paciente» (este último ítem añadido a la escala modificada SERVQHOS en nuestro estudio), siendo ambos muy valorados en relación con la satisfacción.

Los resultados de las preguntas dicotómicas del segundo bloque fueron altamente satisfactorios. Prácticamente la totalidad de los pacientes encuestados $(98,3 \%)$ recomendarían este hospital, muy pocos contestaron tener dudas y no hubo nadie que no recomendará el hospital [17]. El 97,5\% de los pacientes contestaron que no se les realizó ninguna prueba o intervención sin su consentimiento y el $92,4 \%$ afirmaron haber recibido suficiente información sobre su enfermedad [21-23]. Por otra parte, sólo un 4,2\% de los pacientes opinaron no haber estado ingresados en el hospital el tiempo suficiente. Por último, se valoró también la relación interpersonal entre el paciente y el equipo sanitario, para lo cual se les preguntó sobre si conocían el nombre de los profesionales que les atendieron. El 60,5\% afirmó conocer el nombre del médico que les atendió habitualmente, pero en el caso del personal de enfermería, un $69,7 \%$ de los pacientes no conocía el nombre de las enfermeras que lo atendieron, aspecto que coincide con los publicados en otros estudios. Este dato podría ser fácilmente explicable ya que, aunque todo el personal sanitario se presente la primera vez que contacta con el paciente, es más fácil recordar un sólo nombre, el del médico, ya que las enfermeras cambian por turno y día, lo que hace difícil identificar a una enfermera de referencia $[7,14,18,20,22]$.

En nuestro estudio, a diferencia de otros [7], sólo se encontró significación estadística en la variable edad al estudiar su influencia en el nivel de satisfacción global percibida, aunque algunos estudios indican que la relación entre las variables sociodemográficas y la satisfacción son 


\section{VALORACIÓN DE LA SATISFACCIÓN DE LOS PACIENTES INGRESADOS EN OTORRINOLARINGOLOGÍA MEDIANTE LA ESCALA SERVQHOS PISÓN-CÁRCAMO E y DÍAZ DE CERIO-CANDUELA P}

extremadamente pequeñas $[9,22]$. Se realizó un segundo análisis tras agrupar el resto de las variables sociodemográficas, siendo la edad el factor que en mayor medida determina el comportamiento de la satisfacción global del paciente, siendo las personas jóvenes más exigentes en cuanto a la atención recibida [21]. De forma general, se observó que las personas que se encontraron más satisfechas fueron aquellas con una edad comprendida entre los 46-77 años, hombres, casados, sin ocupación laboral y con un nivel de estudios superiores.

Por último, se procedió a valorar las variables sociodemográficas de los pacientes relacionadas con cada aspecto asistencial del primer bloque de respuestas (19 ítems), ambas recodificadas, para agrupar los datos y alternativas de respuestas. A diferencia con otros estudios [20], se encontraron diferencias significativas en la variable demográfica sexo con respecto a los ítems relativos a la calidad subjetiva: «la capacidad del personal para comprender sus necesidades», «la amabilidad del personal con el trato con la gente» $\mathrm{y}$ «el trato personalizado que ha recibido». En todas ellas las mujeres puntuaron mejor estos ítems respecto a los hombres, contestando estar más satisfechas de lo esperado. En cuanto a la variable estado civil, se observaron diferencias significativas con respecto al ítem relativo a la calidad objetiva: «el tiempo de espera para ser atendido por el personal sanitario, donde los casados puntuaron mejor este ítem con respecto al de los no casados.

Al final de la encuesta se dejó un espacio en blanco para sugerencias y para conocer algún aspecto que no estuviera recogido en el cuestionario. Fue cumplimentado por 32 personas. En general, los comentarios recogidos fueron de agradecimiento y opiniones positivas para el personal sanitario y el servicio en general, y alguna sugerencia de mejora del funcionamiento del hospital.

El uso de las encuestas para valorar el nivel de satisfacción es una herramienta sencilla y eficaz, pero presentan algunas limitaciones que pueden influir en el resultado del estudio. Algunos sesgos que podemos señalar en nuestro estudio son: sesgo de confusión (propio del SERVQHOS) por equivocación de algunos ítems que son similares; y sesgo de subjetividad porque la satisfacción del paciente depende de las expectativas y perspectiva de cada persona.

\section{CONCLUSIONES}

La participación en la cumplimentación de la encuesta de satisfacción fue del 63,6\%. La mayoría de los pacientes se mostraron satisfechos en las dimensiones exploradas y casi la totalidad recomendarían este hospital. Se evidenció que la satisfacción está muy ligada a cualidades y competencias del personal en su relación con el paciente. Basándose en los resultados de este estudio y en las opiniones de los pacientes, se pueden incrementar el tamaño muestral para posteriormente proponer una serie de actividades para intentar mejorar las áreas peor valoradas.

\section{DECLARACIÓN DE INTERESES}

Este artículo está basado en el trabajo de fin de Máster Universitario en Metodología de las Ciencias del Comportamiento y de la Salud (Interuniversitario UNED-UCM-UAM) por la Universidad Autónoma de Madrid; la Universidad Complutense de Madrid y la Universidad Nacional de Educación a Distancia de Estela Pisón-Cárcamo presentado en septiembre de 2017 y codirigido por el Dr. P. Díaz de Cerio.

\section{BIBLIOGRAFÍA}

1. Caminal J. La medida de la satisfacción: un instrumento de participación de la población en la mejora de la calidad de los servicios sanitarios. Rev Calid Asist. 2001;16(4):276-9.

2. Durán AG, Durántez FD, Robles JM, Becerra AC, Monchul JV, Ferreras ID. Índices de satisfacción y calidad percibida en una unidad de cirugía mayor ambulatoria de un hospital de tercer nivel. Rev Esp Enferm Dig. 2003;95(12):851-6. 


\section{VALORACIÓN DE LA SATISFACCIÓN DE LOS PACIENTES INGRESADOS EN OTORRINOLARINGOLOGÍA MEDIANTE LA ESCALA SERVQHOS PISÓN-CÁRCAMO E y DÍAZ DE CERIO-CANDUELA P}

3. Sánchez Legrán F. La participación de los usuarios en la sanidad debe ser ya una realidad. Rev SAS Información. 2000;4:3.

4. European Foundation for Quality Management. Modelo EFQM de Excelencia. Madrid: Club Gestión de la Calidad, 1999.

5. Arcelay A., Lorenzo S., Bacigalupe M., Mira JJ, Palacio F., Ignacio E., et al. Adaptación de un modelo de gestión de calidad total al sector sanitario. Rev Calid. Asist. 2000;15:184-91.

6. III Plan de Salud de La Rioja 2015-2019 [Internet]. España: Riojasalud; 2017 [citado 3 agosto 2017]. Disponible en: https://www.riojasalud.es. Noticias.

7. García-Aparicio J, Herrero-Herrero J, CorralGudino L, Jorge-Sánchez R. Calidad percibida por los usuarios de un servicio de Medicina Interna tras 5 años de aplicación de una encuesta de satisfacción. Rev Calid Asist. 2010;25(2):97-105.

8. Monteagudo $\mathrm{O}$ et al. Aplicación hospitalaria del SERVQHOS: factores asociados a la satisfacción e insatisfacción. Rev Calid. Asist. 2003;18(5):263-71

9. Mira JJ, Aranaz J, Rodríguez-Marín J, Buil JA, Castell M, Vitaller J. SERVQHOS: un cuestionario para evaluar la calidad percibida de la asistencia hospitalaria. Medicina Preventiva. 1998; IV:12-8.

10. Parasuraman A, Zeithaml V, Berry L. A Conceptual Model of Service Quality and Its Implications for Future Research. Journal of Marketing. 1985;49(4):41.

11. Mira JJ, Aranaz J. La satisfacción del paciente como una medida del resultado de la atención sanitaria. Medicina Clínica. 2000;114 (Supl 3):26-33.

12. Cadotte ER, Woodruff RB, Jenkins R L. Expectations and norms in models of consumer satisfaction. Journal of marketing Research. 1987;305-14.

13. Oliver RL. A cognitive model of the antecedents and consequences of satisfaction decisions. Journal of marketing research. 1980; 460-9.

14. Arrébola PA, Tejido SÁ, Jiménez AE, Medina PJ, Pérez CS, Guerrero RF, Díaz GR. Estudio de satisfacción en pacientes hospitalizados en un Servicio de Urología. Arch Esp Urol. 2014;67(7):621-7.

15. Marenco-Arellano V, Ferreira L, Ramalle-Gómara E, Crespo A, Rupérez A, Fraile E. Valoración de la satisfacción materna con la analgesia epidural para el control del dolor del trabajo de parto. Rev. Calid. Asist. 2017;32(3):166-71.

16. De la Fuente RA, Jesús FLM, Hoyos VY, León RC, Zuloaga MC, Ruiz GM. Servicio de urgencias de atención primaria. Estudio de calidad percibida y satisfacción de los usuarios de la zona básica de salud Altamira. Rev. Calid. Asist. 2009;24(3):109-114.

17. Jorge-Cerrudo J, Ramón J, Goday A, González S, Sánchez P, Benaiges D. Estudio descriptivo de la mejora de la calidad percibida en los pacientes sometidos a cirugía bariátrica tras la implantación de una vía clínica. Rev. Calid. Asist. 2012;27(4):226-32.

18. Carvajal BJ, García SS, Márquez AM, Hernández LI, Martín-García AM, Cerquella HC. Valoración de la satisfacción de los pacientes intervenidos de vesícula biliar por laparoscopia en un servicio de cirugía general. Rev. Calid. Asist. 2008;23(4):164-9.

19. Mira J, Buil J, Aranaz J, Vitaller J, Lorenzo S, Ignacio. ¿Qué opinan los pacientes de los hospitales públicos? Análisis de los niveles de calidad percibida en cinco hospitales. Gaceta Sanitaria. 2000;14(4):291-3.

20. Braña MB, Carrera MD, De la Villa SM, Avanzas FS, Gracia CM, Vegas PE. Factores sociodemográficos y satisfacción con la atención recibida en mujeres con cáncer de mama. Estudio multicéntrico. Rev. Calid. Asist. 2012;27(1):30-7.

21. Hernández MM, Ochando GA, Mora CJ, Lorenzo MS, López RK. Satisfacción del paciente en una unidad de hemodiálisis: Objetivo de calidad asistencial en enfermería. Revista De La Sociedad Española De Enfermería Nefrológica. 2005;8(2):90-6.

22. Alfonsín SC, Viña VJ. Grado de satisfacción en el paciente trasplantado de pulmón. Rev. Calid. Asist. 2007;22(1):21-7.

23. Hall J, Dornan M. Patient sociodemographic characteristics as predictors of satisfaction with medical care: A meta-analysis. Social Science \& Medicine. 1990;30(7):811-8. 
